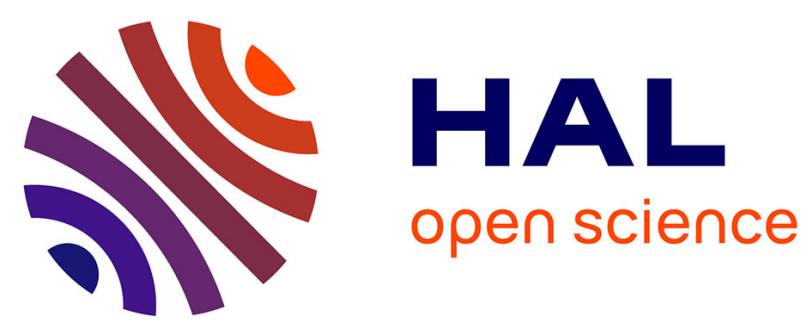

\title{
Materials and process selection from teaching to research and back: the Rhone Alpes experience
}

Luc Salvo, Yves Bréchet, Jean-Yves Buffiere, Didier Landru

\section{To cite this version:}

Luc Salvo, Yves Bréchet, Jean-Yves Buffiere, Didier Landru. Materials and process selection from teaching to research and back: the Rhone Alpes experience. Advanced Engineering Materials, 2001, 3 (8), pp.523-530. 10.1002/1527-2648(200108)3:8\%3C523::AID-ADEM523\%3E3.0.CO;2-Y . hal-01668828

\section{HAL Id: hal-01668828 \\ https://hal.science/hal-01668828}

Submitted on 21 Jan 2022

HAL is a multi-disciplinary open access archive for the deposit and dissemination of scientific research documents, whether they are published or not. The documents may come from teaching and research institutions in France or abroad, or from public or private research centers.
L'archive ouverte pluridisciplinaire $\mathbf{H A L}$, est destinée au dépôt et à la diffusion de documents scientifiques de niveau recherche, publiés ou non, émanant des établissements d'enseignement et de recherche français ou étrangers, des laboratoires publics ou privés.

\section{다)(1) $(5$}

Distributed under a Creative Commons Attribution - NonCommerciall 4.0 International 


\title{
Materials and Process Selection from Teaching to Research and Back: the Rhone Alpes Experience**
}

\author{
Luc Salvo, ${ }^{*}$ Yves Bréchet, Jean-Yves Buffière, and Didier Landru
}

The development of systematic methods for materials and process selection is a relatively new field. The variety of materials and processes available to the engineer, as well as the complexity of the set of requirements encountered in industrial situations, are the driving forces behind the development of software aiming at the guidance of the designer. Born as pedagogical tools, such software, tailored for a given class of materials or of applications, has now in certain cases reached a degree of specialization that is mature and applicable to the industrial design process.

\section{Introduction}

Materials and process selection are key issues for highperformance design. The variety of materials and processes available to the engineer (more than 80000 materials, more than 8000 processes) has led to what is known as "over choice" in the design process. At the same time, cost considerations, tradition, and the natural inhibition about new choices that might lead to unexpected problems, are strong incentives for conservative solutions. Training engineers in materials and process selection presents the challenge of opening their minds to innovative solutions, at the same time as making them aware of the value of accumulated experience. Developing systematic procedures for materials and process selection in mechanical design has been undertaken by a number of teams, following the pioneering work of Prof. M. F. Ashby. ${ }^{[1]}$ In the Rhone-Alpes Region of France, mainly in Grenoble and Lyon, this activity has two closely related aspects: teaching and research. The purpose of this review is to give a summary of the work performed in this field, concentrating on the strong coupling between these two aspects: the tools developed in research are used in teaching, and the projects done by the students serve as test cases to drive further development in research. A detailed reference list provides sources of further information in this emerging field.
[*] Dr. L. Salvo

GPM2, INP Grenoble

BP46, 38402 Saint Martin d'Heres (France)

Dr. Y. Brechet, Dr. D. Landru

LTPCM, INP Grenoble

BP75, 38402 Saint Martin d'Heres (France)

Dr. J. Y. Buffière

GEMPPM, INSA Lyon

Avenue A. Einstein, 69621 Villeurbanne (France)

[**] All the work presented in this paper was performed with the help of many students: David Bassetti, Nicolas Demarty, Jose Deocon, Lindo Duratti, Yannick Guillanvarch, Gustav Heiberg, Christophe Lebacq, Vincent Lopez, Ludovic Lae, Paul Lemoine, Pierrick Pechambert. Numerous discussions with colleagues or people from industry are gracefully acknowledged: Eric Andrieu, Bernard Baroux, Hervé Biausser, Francois Cansell, David Cebon, Jean Charbonnier, Alexis Deschamps, Dominique Duly, Michel Dupeux, David Embury, Jean Etourneau, Roger Fougères, Lindsay Greer, Didier Imbault, Sylvain Jacob, Alain Jantzen, Thierry Jeggy, Jean Marie Maldjian, Gérard Maeder, Guy Murry, Jean Philibert, Christine Ponthieu, Daniel Quenard, Jean Hubert Schmitt, Hugh Shercliff, Muriel Véron, Alain Vincent. Continuing inspiration from Mike Ashby is at the root of this work. This project was partially funded by the Korber foundation. 


\section{Systematic Materials and Process Selection Methods}

Systematic methods for materials and process selection have only recently been developed to a point where they can be used in an efficient manner in the design procedure. This progress is partly due to increased computer power and the development of user-friendly interfaces allowing a direct and iterative treatment of multicriteria selection problems. Even if the core of the method is not a computer program, computer implementation is a useful test for evaluating the practical efficiency of the proposed concepts. In any method for indus- trial design, one has to separate the method from its implementation. The underlying methodology of the general approach developed in Cambridge, further referred to as the "Ashby Method", can be summarized as follows. ${ }^{[1,2]}$

- A hierarchical structure for the databases: both materials and processes are organized in a systematic manner, from very general databases to more specific ones, with the accuracy of the data increasing with the level of specificity of the database.

- A description of all the members of a database by a set of equivalent attributes allowing comparison. No data should be missing (which leads to a need to estimate attri-
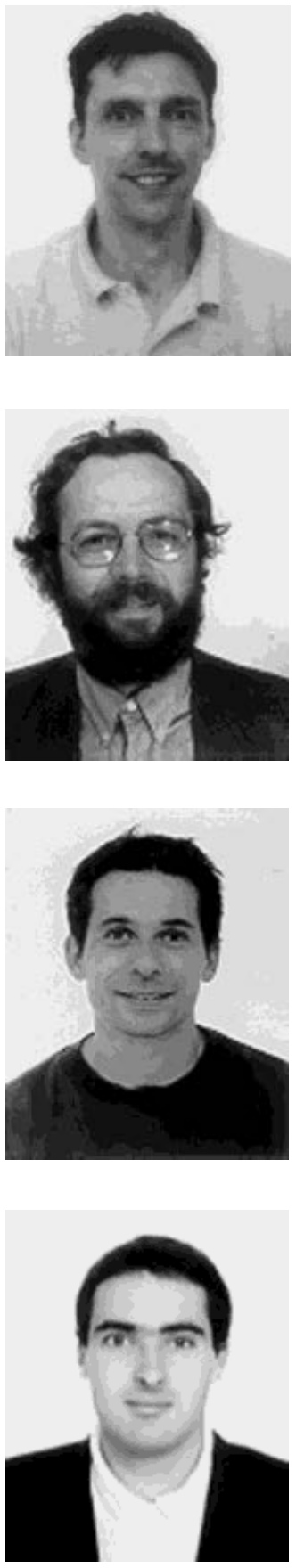

Jean Yves Buffière is a lecturer at INSA Lyon where he teaches Materials Science and Materials Selection within the Industrial Engineering Department. His main interest in research is the study and modelling of plasticity and damage of metallic micro-heterogeneous materials during monotonic or cyclic deformation. During the last five years, in collaboration with the European Synchrotron Radiation Facility in Grenoble (ESRF) he has been working on the application of high resolution X-ray tomography to the characterisation of structural materials.

Yves Brechet is professor in Materials Science at Institut National Polytechnique (INP) de Grenoble. His research interests, mainly in modelling, concern phase transformation in metals and alloys, microstructure/mechanical properties relationships, and materials and process selection methods.

Luc Salvo is a lecturer at INP Grenoble where he teaches Materials Science and Materials Selection. His main interest in research are materials and process selection and the study of relationship beteween microstructure and mechanical properties (in semi-solid materials, metal foams and superplastic alloys). Microstructure is characterised using high resolution X-ray microtomography in collaboration with the European Synchrotron Radiation Facility in Grenoble (ESRF).

Didier Landru made his PhD at INP Grenoble in Materials and Process Selection. His work lead to the development of several software: a first one which takes into account shape, material, process and joining in the selection of a component, a second which was a first attempt to developp surface treatment selection and a last one that allow to screen potential applications for a new material. He is now an engineer in the ST Microeletronics group. 
butes). No material or process class should be over- or under-represented.

- An objective evaluation of performance, relying on the methodology of performance indices for materials selection, and of simple cost estimation for process selection. ${ }^{[1,3]}$

- A deductive approach aiming at minimizing the need for judgements and subjective criteria. This requires a strict definition of the constraints (which have to be fulfilled) and of the objectives (which have to be optimized).

- A progressive approach: screening the possible candidates (by process of elimination), ranking them according to their ability to optimize the objective, and then getting the necessary further information on a limited number of possible candidates.

This general framework was first applied to materials selection. ${ }^{[1]}$ Then a similar strategy was applied to process selection, and more recently the same methodology has been implemented in the CES (Cambridge Engineering Selector) software $^{[3]}$ in order to couple materials and process selection, through a systematic use of links between databases. These methods were first applied to simple problems, involving situations with single constraints and single objectives. Multiple constraint situations were then included using the coupled equation methods, allowing the dominant constraints to be identified in simple situations. Multiple objective situations have been dealt with using simple approaches of value analysis. These improvements to the initial approaches have enabled the method to be applied to cases closer to industrial situations where the multiple-criteria nature of the selection process is central. Currently the databases included in the software comprise more than 2000 materials. Both the methodology of "performance indices" and the use of this software are the core of teaching in selection methods now currently implemented in materials science and engineering studies in Rhone Alpes.

\section{Teaching Materials Selection in Lyon and Grenoble}

At INSA Lyon, courses on material selection are given in the Materials Science and Engineering Department, and in the three other departments that include some knowledge of material science in their courses: Industrial Engineering, Mechanical Engineering, and Energy and Environmental Engineering. Different teaching methods have been adopted according to the importance given to materials science in the whole syllabus of the department. In the Industrial Engineering Department, these courses are given to first-year students who receive a basic course on materials at the same time. While getting familiar with classes of materials and their properties, students use selection maps and performance indices during exercises. During a $4 \mathrm{~h}$ practical session groups of three students select a material for a given product with the help of the Cambridge Materials Selector software. Through this demonstration, students discover the content of a material database (i.e., what kind of data they can find in such databases, and what is missing) and how they can use it efficiently. The situation is almost identical in the Energy and Environmental Engineering Department, where students receive a $20 \mathrm{~h}$ basic course on materials with an emphasis on the use of material databases, thermal properties mainly, through the use of CMS. In the Mechanical Engineering Department, selection methods are taught via micro-projects. Groups of three or four students are asked to select a material and a process (Cambridge Process Selector) for a manufactured product (a different product for each group). At the end of the project $(12 \mathrm{~h})$ the solutions are presented and discussed collectively. In the Materials Science and Engineering Department, $4 \mathrm{~h} \mathrm{tu}$ torials are carried out to introduce performance index and selection maps. Finally, a $15 \mathrm{~h}$ course is devoted to materials selection at post-graduate level. Although the students involved have already received detailed courses on polymers, ceramics, metals, and composites, those tutorials are usually their first opportunity to consider and compare all materials classes for a single design problem. Solutions for multiple constraints and/or compound objectives selections are described. A $4 \mathrm{~h}$ practical at the end of the course illustrates the possibilities of the CMS software (with an insight into more and more precise databases) and also its limits: no weight factors for selection criteria, no ranking of the selected materials.

At Institut National Polytechnique de Grenoble, the emphasis has been upon project work on materials and process selection problems. Training in materials and process selection is a central topic in the curriculum of "structural materials engineers": these students have a basic training in materials science, mechanics, or physics, and for 2 years they have a common curriculum with project-based learning for half of the teaching. The students receive an extended course in materials and process selection $(30 \mathrm{~h})$ followed by a project, supplied by industry, which allows them both to apply materials and process selection methods, and to find their limitations on real industrial problems. In this project, co-tutored by an engineer in industry and a professor, they have to select materials or processes (or both), or to find applications for a material, or to find a replacement for an existing material. It is a standard comment on the materials and process selection methods that they mainly addressing teaching purposes and that the complexity of industrial problems is out of reach of these approaches. To test this assertion, to identify the possible limitations of the method, and to try to overcome them the method is applied by students to real industrial cases.

The advantage of doing this is double: one can see how far "inexperienced engineers" can go with the systematic approaches, and one can see the directions where further development is needed. The time spent on the projects by each group of three students is about $50 \mathrm{~h}$. The companies setting the projects range from the very small (one person) to the very large (multinational companies). The fields of activity range from transformation industries to manufacturing and service industries. Examples from aerospace, automotive, 
and chemical industries, and sports equipment manufacture are to be found in the 200 case studies that have been undertaken in the last 6 years. It cannot be reasonably expected that final-year engineering students, even with the help of a tutor in industry and a tutor at the university, will be able to treat completely a materials or process selection problem. But the approach of not pre-selecting the case studies for these projects, and having them analyzed by inexperienced designers, allowed a fair overview of the possibilities of the selection methods and their implementation software. Some of these case studies have been published. ${ }^{[3]}$ From this experience, in $50 \%$ of the cases the systematic selection procedure provided a method of identifying possible solutions, and the proposed solutions were reasonable ones for which further information was worth finding.

However, the problem of giving different weights to the different criteria was difficult to deal with. The reason for this difficulty was that the extra information (on dimensioning, value analysis) allowing the application of the objective multicriteria methods is difficult to obtain. It appeared that in about $25 \%$ of the cases the selection problems were obviously closely related to secondary processes such as joining and surface treatments, and could not be dealt with accurately with the existing tools, but required the development of specialized methods. The problem with the existing tools was that the information concerning these secondary processes appeared to be not sufficient to be really selective. In the remaining $25 \%$ of the cases, the method was unable to provide a meaningful approach to the problem. These cases were often related to finite life design, for example in relation to corrosion or wear, where the life expectancy of the component was the crucial issue. Both the lack of information, and the lack of a systematic selection method for this kind of problem, limited the efficiency of the procedure. The main difficulties started very early in the procedure, in the definition of the set of requirements: in selection problems, it very rapidly becomes clear that an accurate and objective definition of the requirements is both crucial and difficult. The second difficulty came with the problem of multi-criteria selection: the objective methods $s^{[1,3]}$ require information that is sometimes difficult to obtain. The third difficulty appeared when coupling between process and materials selection was so strong that a simple two-way link relationship was not sufficient to describe it. Last but not least, several case studies led to the selection of multi-material structures, or even of pre-defined products rather than materials. This "experimental investigation" of the effectiveness and limits of the "Ashby Method" motivated further research in the methodology of materials and process selection.

\subsection{Example of a Student Project: Materials Selection for a Satellite Cryostat}

In a satellite, a detector is connected to a cryostat via a rope whose function is to convey heat without transmitting vibrations. Since the detector and the cryostat are to be launched with the satellite, minimum mass is an objective. Ensuring a prescribed heat transfer with a minimum mass leads to the minimization of $\lambda / \rho$, where $\lambda$ is heat conduction, and $\rho$ the density. Ensuring maximum flexibility leads to a multifiber structure and the best material maximizes $\lambda^{2} / E$ where $E$ is the Young's modulus. The material selection becomes metals or graphite. On the metallic side, pure aluminum, pure copper, and pure silver are possible solutions. Aluminum is better if mass is the major concern, silver is better if the main concern is to avoid transmitting vibrations. Graphite is an excellent solution if mass is a central concern, but degassing might be a problem: pre-annealing is probably required. To minimize emittivity, it is also useful to think of a metallic packaging for a graphite cable, but at the expense of mass minimization. This problem was very suited to a performance index approach, and the properties required in the selection were available in the databases. However, the project is not a simple exercise of applying the computer software: analysis of the possible solutions, of the joining of the connector, or the possible degassing effects is part of the project. A number of case studies can be treated using the general methods. ${ }^{[1,2]}$ The next example shows the limitations of the methods.

\subsection{Another Example of a Student Project: Material Selection for Latent Heat Thermal Energy Storage}

Domestic boilers may be designed for storing thermal energy during the night, when electrical power is cheaper. An electrical resistance heats the water that will be used during the day. The amount of thermal energy that can be stored by this method can be increased by introducing a latent heat thermal energy system composed of a material in a container. This material undergoes a phase transformation (solid to liquid transformation generally) when the water is heated to about $65^{\circ} \mathrm{C}$ at night. Through this phase transformation some heat is stored in the boiler (latent heat of fusion) and can be recovered the day after when the temperature of the surrounding water decreases. A large value of the heat of fusion per unit volume or per unit mass is of course a primary requirement for the material that undergoes the solid/liquid transformation but other properties listed below need to be taken into account:

- high specific heat,

- melting point appropriate for the application (about $\left.65^{\circ} \mathrm{C}\right)$,

- chemical stability and little corrosion (container),

- not hazardous, inflammable, or poisonous,

- reproducible crystallization with no degradation during the heating and cooling cycles,

- little supercooling,

- high density,

- high thermal conductivity in solid phase,

- high diffusivity/low viscosity,

- low thermal expansion during the phase transformation, and

- low cost. 
From the literature, it appeared that potential materials for latent heat storage can be divided in three groups: inorganic compounds (mainly hydrated salts), organic compounds (paraffins, fatty acids), and eutectics of organic or inorganic compounds. A detailed study was undertaken to investigate which material would be best suited for increasing the stored thermal energy by $50 \%$ with no change in the boiler volume or shape. A first set of 49 materials with melting points between 45 and $80^{\circ} \mathrm{C}$ was selected from the literature. Properties like the toxicity, viscosity, and supercooling were not available for many of those materials thus, in a first step, a ranking was performed using the known (mainly numerical) properties. Three methods were used.

A first merit index $I$ of the material was defined:

$\alpha$ and $\beta$ are two weight factors, $A$ and $B$ are two properties that have to be maximized or minimized, respectively.

$I=\alpha\left(A / A_{\max }\right)+\beta\left(B / B_{\min }\right)+\ldots$

An improvement of this equation can be obtained by considering the variation range of the property instead of absolute values.

$I^{\prime}=\alpha^{\prime}\left(A-A_{\min } / A_{\max }-A_{\min }\right)+\beta^{\prime}\left(B_{\max }-B / B_{\max }-B_{\min }\right)+\ldots$

For calculating $I$ and $I^{\prime}$ the following properties were taken into account: melting point, latent heat of fusion, specific heat, thermal conductivity, thermal diffusivity, and thermal expansion coefficient. Two sets of weight factors were used and compared. For both methods, the main difficulty lay in the determination of the weight factors. Therefore, a third merit index was established by calculating only the ability of the material to store thermal energy through its specific heat (liquid and solid) and its latent heat of fusion.

The first 12 materials obtained by each of the three methods were selected, which resulted in 21 materials. A second selection stage was then performed by considering a yes/no criterion on non-measurable properties such as toxicity and corrosion. From this analysis, it turns out that mineral salts can store a lot of thermal energy, which makes them attractive but they are ruled out by supercooling, toxicity, or corrosion problems. Organic compounds cannot store as much thermal energy as mineral salts but their thermal, physical, and chemical stability makes them attractive. Finally, the lack of reliable data on the ability of eutectic compounds to store energy eliminated a large number of such materials. A small subset of five materials comprising at least a representative of each class was selected. In a further step in the selection process, precise experimental measurements of some properties (viscosity, thermal expansion, latent heat of fusion) were carried out in the laboratory. Some attention was also paid to the container material and its possible interaction with the phasechange material.

In this case study, the difficulty was mainly finding information on materials that are not available in the current data- bases. Such situations led the students to perform a literature search, internet investigations, and expert interviews, in order to go further in their selection procedure.

\section{Research Activity in Materials and Process Selection Methods in Rhone Alpes}

Research activity in this field has been performed in close collaboration with the team in Cambridge. ${ }^{[4]}$ From the industrial case studies, it was decided to investigate the aspects of materials and process selection that were imperfectly treated by the generic methods. The activity therefore developed in three directions.

- Developing methods to deal with the multi-criteria aspect as well as with the iterative nature of the selection process. This was aimed at implementing in real situations the methods for multiple constraint and multiple objective selection, using pre-dimensioning of components and estimating the value coefficients. The difficult problem of criteria of unequal importance was dealt with using the technique of "fuzzy logic". The iterative nature of selection is guided by a simple expert system.

- Developing techniques, less generic but perhaps more applicable when the selection problem is becoming more specialized. At this level a special interest was devoted to situations requiring a strong coupling between materials and process selection. The general methodology developed for these questions relies on a "pre-defined questionnaire" approach rather that on the "open ended" approach proposed in the generic method. Examples of this approach concern the selection of joining methods, surface treatments, and the selection of cast aluminum alloys.

- Using the selection techniques as tools for materials or multi-materials development for a specific application. This problem is illustrated here for selection of polymer matrix composites and for optimal design of sandwich structures: two problems whose numerical complexity has led to the development of special optimization methods using genetic algorithms.

The integration of all the methods dealing with multicriteria selection has been done in the CAMD (Computer Aided Mechanical Design) software. ${ }^{[5-7]}$

The first problem, multiple constraint design, can easily be solved by a systematic pre-dimensioning of the component, provided a simple geometry and simplified loading conditions are prescribed. After the user has chosen the loading modes and the design constraints of the component, a systematic check through all of the constraints identifies all the data that are required, and the limiting constraints can be found objectively. The user is prompted to provide the missing information. This is nothing but a systematic and automated implementation of the "coupled equation method" in order to identify the limiting constraints as far as possible with the existing information. Naturally, this procedure requires that the relationships are known between the design 
element's properties and the behavior of the part under external loads. Standard solutions for mechanical and thermal loading in simple geometries are stored in a database of "component behavior". For instance, the deflection of a beam under a distributed load will be recorded in the program. The limiting constraints will be identified if and only if all the necessary information is available. If not, the two selection criteria will be treated separately. For instance, if a component loaded in compression has to resist both elastic buckling and plastic deformation, the multiconstraint selection requires knowledge of the load and the length of the component. If both are known, a coupled equation method is implemented. If one piece of information is missing, the two constraints are treated separately leading to a couple of performance indices. This treatment of the multiple constraint problem is fully objective, since it relies only on the expressed requirements.

The second problem, identifying exchange coefficients, has been treated by the analysis of existing solutions of given design problems. The underlying idea is that existing solutions somewhat reflect the relative importance of various criteria. One might find in the aerospace industry carbon-fiber connecting rods, as well as steel ones or light alloys ones. But carbon-fiber connecting rods would not be found in ordinary cars. There is thus information hidden in the choices currently made, but in order to extract this information, one has to make some hypotheses about the coexistence of different solutions. For mature fields, it is reasonable to assume that coexisting solutions are somewhat equivalent for the set of requirements. Equivalence has to be understood in terms of value function, within an admissible dispersion. Coexisting solutions are assumed to have equal value, or alternatively the dispersion in each performance measure for the existing solutions is assumed to correspond to an identical dispersion in the overall value. This allows an estimate of the exchange coefficients between performance measures to be made. The results of this estimation are shown to be consistent with a deductive approach, such as that proposed by a simplified value analysis. The example of various structural components (such as a connecting rod) leads to an estimated exchange coefficient between weight and price of 3 Euros per $\mathrm{kg}$ for cars, 45 Euros per $\mathrm{kg}$ for airplanes, and more than 60000 per $\mathrm{kg}$ for satellites. Estimations from the value of fuel saving, gain in utility load, or cost of launch are in the same range. However this method, implemented in the VCE software, is applicable even when a clear-cut value analysis is not possible, provided design solutions already exist.

The third problem, multiple design elements, needs more explanation. Design elements include the material, the shape, the forming processes required to achieve the shape, surface treatments, the number of components needed to make the product, and the assembly methods for joining those components. Designing a product amounts to choosing the optimal combination of design elements. Each combination that verifies all product design constraints is a valid design solution. The best design solutions are those that maximize design performance. Designing several design elements requires the user to define the kind of solutions being sought. Indeed, optimizing all elements is not always necessary or possible; for instance, when the shape of the product is already prescribed by existing design constraints, or where performing a surface treatment is not useful, or industrially inconvenient. This information is gathered into a design model that gives for each design element the way it is involved in the design: it can be prescribed, not considered, or it has to be optimized. From this "design model", all possible design solutions are created by combining selected elements from different design element databases. Among all the possible design solutions, those that do not fit the set of requirements, or which contain incompatible elements (such as machining a ceramic), are eliminated. This leaves all technically valid solutions for the design. In a final stage, solutions are ranked according to their performance. To prevent combinatorial explosion of the number of solutions to be compared, creation of initial solutions is performed using a recursive algorithm, ranging from low-detail solutions to high-detail ones. Four major "design elements" have been considered: materials, shapes, forming processes, and joining processes. Surface treatments were treated separately as a design element added subsequently to cure a problem associated with fatigue, wear, or corrosion.

The databases used for materials, forming processes and shapes are identical to those developed for CES. The joining processes and the surface treatments have been treated according to a "pre-defined questionnaire".

The fourth problem, naïve as it seems, is nevertheless at the core of the design procedure: no proper design can be made if the set of requirements is not cautiously established. This is by no means a straightforward question, and with the increasing complexity encountered in industrial cases, the need for guidance in this iterative procedure becomes obvious. The set of requirements is the result of the functional analysis of the component. Ideally, if this analysis is properly performed, the set of requirements would be a problem. But the complexity of the real world of design imposes an iterative procedure in the design process: from the results of the selection for a given set of requirements, one is led to modify the set of requirements, to correct errors, to make the requirements more strict or more flexible. It is a strength of the systematic selection procedure to be able to evaluate rapidly the influence of a set of requirements on the possible solutions, and to guide this iterative procedure. Beside the correction of possible errors, the iterative procedure contributes to the overall "hierarchical strategy" outlined in the introduction: the first set of requirements is necessarily crude, and will be refined as the selection procedure proceeds. A clear outcome of the study of industrial case studies is the conjugate evolution of solutions and more and more accurate set of requirements, while the selection becomes more and more focussed. A systematic iterative procedure guides the user to make improvements to the set of requirements, by a repeated analysis 
of the results corresponding to the current state of the set of requirements. The iterative process treats the set of design elements and requirements as a whole, checking their compatibility, and that known solutions are identified among the possible solutions. It is not a real expert system (in the sense that it cannot learn from past experience), but it allows a systematic investigation of the classical errors encountered in defining a set of requirements.

The selection according to the final set of requirements is then performed for multiple design elements, multiple constraint problems, or multiple objective selection as appropriate. The software developed along these lines (CAMD), is an implementation of the various techniques in a way such that the choice remains as objective as possible. It includes the possibility of selecting joining methods and surface treatments at a simplified level: joining methods are selected according to the compatibility between the shape and the materials of the components; surface treatment methods are selected with similar compatibility requirements, along with the functional goal being aimed at by considering a surface treatment. The possibility of defining the requirements not only with strict numbers, but allowing for some flexibility in the requirements (which is the strength of fuzzy logic methods), permits some account to be taken of the trade-off between different requirements.

The whole philosophy of the approach illustrated by CAMD, as well as in the Cambridge Materials Selectors, is an "open-ended questionnaire" that puts basically no limitation on the format of the set of requirements. It allows an evolution of the set of requirements during the selection procedure by an iterative treatment of the problem, but it places no limit on the content of the set of requirements, provided it can be translated in terms of the information available in the databases on materials and processes. Such an approach is very efficient in the first step of the design procedure, but it becomes limited when more detailed and specific design requirements enter into play. In such situations, the generic methods exemplified here can be applied, but they will not be selective: the criteria that allow choice between various joining methods for instance, might be the time allowed after joining before the component can be moved away, or the place where the joining has to be performed. Not surprisingly, this problem appears with its full strength for secondary processes (joining, surface treatments) where the process operates not on a material, but on a component. This situation has led to the development of a different strategy relying on an "expert questionnaire" approach, which will now be discussed. When the processing situation becomes either very specialized or very related to the detailed features of a components, a "pre-defined questionnaire" approach appears to be more selective than selection by attributes. This situation was encountered in three cases of specialized methods: selection of surface treatments, selection of joining methods, and selection of cast aluminum alloys. In each case, a different questionnaire was developed, which cannot be part of a generic one. However, the strategy itself is generic and likely to be applied to other situations in materials and process selection.

A variety of specialized software for selecting materials has been developed, either following the methodology of the "open questionnaire", or following a set of "predefined questions". They are listed in Figure 1. They range from the materials selector to the product selector: they can also be a guide for materials development. The variety of topics treated is a sign of the robustness of the selection methods, the question remaining for each problem is the relative efficiency of the two strategies.

(1) FUZZYMAT: Fuzzy J ogic based Material Selector

(2) CAMD: Integrated Multidesign element selector

(3) ASTEK: Selecting joining methods

(4) FUZZYCAST: Cast Aluminium alloys Selector

(5) STS: Selecting surface treatments

(6) Composite Materials selector

(7) Sandwich Structure Selector

(8) FU/\%YGIASS: Glass composition Selcctor

(9) Selector for Creep Resistant Polymers

(10) Selector for Building Thermal Insulator

Fig. 1. Materials and process selection software developed in the Rhone-Alpes region $[4,8-16]$.

\subsection{Further Research}

While becoming more specific, the method loses in elegance and generality, when compared to the simple "Ashby Meth$\mathrm{od}^{\prime}$, but this is the price paid for coming closer to capturing the almost endless variety of selection requirements in realistic situations. However, even if the details of the method cannot always be applied directly to the specific cases investigated above, the general framework of structuring the databases and the selection procedure appear to offer reliable guidelines to enable progress from a useful pedagogical tool to a design tool that might be applicable to industrial problems.

Further developments in the field are certainly expected and needed in order to improve the applicability of the method. Among the possible routes to be explored, one can list:

- development of a systematic tool to search for applications for a given material,

- development of "rule based" systems for selecting for a finite lifetime (corrosion, wear, fatigue), and

- development of a systematic procedure to deal with multimaterial selection, or with the strategy of decoupling functions.

Other aspects of industrial relevance are under investigation by the Cambridge team, such as "aesthetics in design", or "ecodesign". A generic problem in design, at the point reached by the tools currently available, is their integration with finite element codes in the various steps of dimensioning. Another strategy suggested by the difficulties of choosing materials and processes is to develop tools relying on 
case-based learning in order to guide the selection procedure. This strategy is by nature conservative, but allows storage of existing experience in a reusable manner. It is likely that further developments in the field of materials and process selection methodologies will have to conjugate both approaches, the fully deductive one exemplified in this paper, and the inductive one relying on expertise storage. The conceptual tools involved in this field are far beyond materials science and process engineering; they make use of advanced computer science, artificial intelligence, and complex optimization techniques.

\section{Conclusions}

The development of systematic methods for materials and process selection is a relatively new field. The variety of materials and processes available to the engineer, as well as the complexity of the set of requirements encountered in industrial situations, are the driving forces behind the development of software aiming at the guidance of the designer. Born as pedagogical tools, such software, tailored for a given class of materials or of applications, has now in certain cases reached a degree of specialization that is mature and applicable to the industrial design process. More work is needed to proceed further toward a better integration of the non-technical, but nevertheless crucial, aspects of engineering design.

[1] M. F. Ashby, Materials Selection in Mechanical Design, Butterworth Heinemann, Oxford 1999.

[2] Y. Brechet, M. F. Ashby, L. Salvo, in Sélection des matériaux et des procédés de mise en oeuvre, in Traité des Matériaux, Vol. 20 Presses Universitaires de Lausanne, 2001.
[3] Granta Design Ltd, Cambridge selection software: CMS 1995; CPS 1997; CES 1999, Cambridge.

[4] Y. Brechet, D. Bassetti, D. Landru, L. Salvo, Prog. Mater. Sci. in press

[5] D. Landru, Y. Brechet, CAMD software, Grenoble 1999.

[6] D. Bassetti, PhD Thesis, Institut National Polytechnique de Grenoble 1998.

[7] D. Landru, PhD Thesis, Institut National Polytechnique de Grenoble 2000.

[8] C. Lebacq, T. Jeggy, Y. Brechet, L. Salvo, Materiaux et Techniques 1998, 5, 39.

[9] Y. Brechet, D. Bassetti, P. Pechambert, Mater. Sci. Forum 1996, 435, 121

[10] A. M. Lovatt, D. Bassetti, H. R. Shercliff, Y. Brechet, Int. J. Cast Metals Res. 1999, 12, 211.

[11] D. Bassetti, Y. Brechet, G. Heiberg, I. Lingorski, A. Jantzen, P. Pechambert, L. Salvo, Materiaux et Techniques 1998, 5, 31 .

[12] P. Pechambert, D. Bassetti, Y. Brechet, L. Salvo, in Proc. 7th Int. Conf. Composite Materials (ICCM7), Institute of Materials, London 1996, p. 283.

[13] D. Bassetti, Y. Brechet, G. Heiberg, I. Lingorski, P. Pechambert, L. Salvo, in Euromech Conference on Sandwich Structures, St Etienne 1999, p. 221.

[14] D. Bassetti, Y. Brechet, G. Heiberg, I. Lingorski, P. Pechambert, L. Salvo, in Proc. Conf. Composite Design for Performance, (Ed.: P. Nicholson), Lake Louise 1998, p. 88.

[15] C. Lebacq, Y. Brechet, T. Jeggy, L. Salvo, H. R. Shercliff, submitted to Materials and Design, 2001.

[16] J. Deocon, L. Salvo, P. Lemoine, D. Landru, Y. Brechet, R. Leriche, in Metal Foams and Porous Metal Structures (Eds: J. Banhardt, M. F. Ashby, N. A. Fleck), MIT Verlag, Bremen 1999, p. 325. 